\author{
Professor Natalja KOSAREVA, PhD \\ E-mail: natalja.kosareva@vgtu.lt \\ Professor Aleksandras KRYLOVAS, PhD \\ E-mail: aleksandras.krylovas@vgtu.It \\ Professor Edmundas Kazimieras ZAVADSKAS, PhD \\ E-mail: edmundas.zavadskas@vgtu.lt \\ Vilnius Gediminas technical university, Lithuania
}

\title{
STATISTICAL ANALYSIS OF MCDM DATA NORMALIZATION METHODS USING MONTE CARLO APPROACH. THE CASE OF TERNARY ESTIMATES MATRIX
}

Abstract. One of the most important stages of solving MCDM problems is normalization of initial decision-making matrix. The impact of 5 widely used normalization methods on the best alternative determination accuracy in the case of ternary estimates decision matrix is analysed in the article. Alternatives ranked by applying SAW method. Monte Carlo procedure was conducted fordata matrices of different dimensions and both optimization directions. Two cases - the more and the less separable alternatives- were analysed.None of the 5 methods were the best or the worst in all cases. Nevertheless, Minmax method inmost cases is significantly better than other. The Log method is the worst in some cases, but it is the best (or one of the best) in other cases. The highest values of the best alternative detection accuracy were accompanied by the lowest standard deviations of experiment results, respectively, the lowest values - by the highest standard deviations.

Keywords: normalization methods, multi-criteria optimization, Monte Carlo method, SAW.

JEL Classification: D81, C44, C63

\section{Introduction}

Multiple criteria optimization methods are being applied in various fields of everyday human activities. Usually we have to solve the task of selection the "best" alternative from the finite or infinite set of alternatives when alternatives are evaluated according to the few criteria. One of the most important stages of solving MCDM problems is normalization of initial decision-making matrix. This procedure is necessary because of different units of measurements of different criteria. Moreover, because of different normalization directions, distinct formulas are being applied for the same normalization method. In this research we will examine the impact of various normalization methods on the best alternative

DOI: $10.24818 / 18423264 / 52.4 .18 .11$ 
Natalja Kosareva, Aleksandras Krylovas, Edmundas Kazimieras Zavadskas

determination accuracy. We limited ourselves with discrete optimization problem solution.

Values of the different quantitative or/and qualitative criteria are aggregated into single criterion value, which is used for the final ranking of alternatives. Such aggregation is possible only for the dimensionless data. Unfortunately, researchers often underestimate the importance of the proper selection of data normalization method for solving specific decision-making tasks. However, normalization techniques have significant impact on the results of decision process and can modify the ranking of alternatives and final decision. The purpose of this research is to compare the accuracy of ranking results of several well known normalization methods applied together with Simple Additive Weighting (SAW).

A comprehensive review of existing normalization methods provided by Jahan and Edwards, 2015. The authors identified 31 normalization method and distinguished these groups of methods: sum-based, linear-ratio-based, linear maxmin dimensionless methods, nonlinear dimensionless methods (z-transformation, etc.) and target-based normalization methods usually applied in medical decisionmaking. The main shortcommings of normalization methods were revealed: some sum-based methods (such as vector normalization) may depend on the evaluation unit (Opricovic and Tzeng, 2004), lack of symmetry in the pair of benefit and cost criteria normalization formulas, rank reversal after adding or deleting alternatives, unability to handle negative values, also, some non-monotonic normalizations have a higher concentration towards the values zero/one. Influence of normalization tools on COPRAS-G method applied for material selection task proposed by Yazdani et al., 2017. The results show, that depending on the number of criteria and number of alternatives material, ranking can be changed when a different normalization tools are considered. In Podviezko and Podvezko, 2015 it is shown that different types of transformation and normalization of data applied to popular MCDA methods, such as SAW or TOPSIS may produce considerable differences in evaluation. In Krylovas et al., 2017 WEBIRA (WEight Balancing Indicator Ranks Accordance), SAW and EMDCW (Entropy Method for Determining the Criterion Weight) methods were compared for 4 different data normalization methods. It was exposed that WEBIRA is the least affected by the data normalization, while EMDCW is the most affected method. In Krylovas et al., 2018 comparative statistical analysis was accomplished for 7 parametric classes of normalization functions in the case of Gaussian distribution of decision making matrix elements.

Review of normalization methods used in construction engineering and management, and their applications there are presented by Kaplinski and Tamošaitienè, 2015. The study of Chakraborty and Yeh, 2009 compares four commonly known normalization procedures in terms of their ranking consistency and weight sensitivity when used with TOPSIS to solve the general MADM problem. The study results justify the use of the vector normalization procedure for TOPSIS and provide suggestive insights for using other normalization procedures 
Statistical Analysis of MCDM Data Normalization Methods Using Monte Carlo Approach. The Case of Ternary Estimates Matrix

in certain decision settings. In Zavadskas et al., 2006 transformation through a normalization of vectors and the linear transformation were compared for TOPSIS method.Research of Celen, 2014 also evaluated the effects of the most popular four normalization procedures on decision outcomes of the TOPSIS method evaluating the financial performances of 13 Turkish deposit banks. The study revealed that vector normalization procedure, which is mostly used in the TOPSIS method by default, generated the most consistent results. In Stanujkicand Zavadskas, 2015 a specific normalization procedure, which introduces a compensation coefficient that better match the decision-maker preferences is proposed.Stanujkicet al., 2017a proposed ARCAS approach is based on the use of the ARAS method, a new normalization procedure, and the SWARA method. Stanujkic et al., 2017b presented the improved Operational Competitiveness Rating (OCRA) method where the original normalization procedure has been replaced by a new one.

The article is organized as follows. Section 2 provides general research scheme -5 normalization methods, two cases of initial data matrices generation procedures. A detailed description of Monte Carlo experiments and their results are given in the Section 3. Section 4 is devoted to conclusions and plans of the future research.

\section{General research scheme}

This research analyses data normalization methods for MCDM tasks when the data is generated by randomly simulating alternative ratings on a three-point scale. In all cases we calculated how many times the best alternative, for which the estimates are generated with predetermined probabilities, was correctly identified. Other alternatives have been generated with equal probabilities. The paper deals with two cases of estimates matrices. In the Case 1 the best alternative had statistically higher estimates, i.e., the probability of an estimate 3 was higher in the direct case and the probability of an estimate 1 was higher in the inverse case. In the Case 2 the best alternative for both direct and inverse optimization has the higher probability of an estimate 2 with equal probabilities of other estimates. So, in the Case 1 the best alternative was better distinguished, while in the Case 2 not very clearly. Generated matrices weren't filtered by rejecting a priori weak alternatives (do not belonging to the Pareto set of solutions). Such filtering would reduce the number of cases when the best alternative was correctly determined. However, this research hasn't the goal to calculate accurately averages of statistical estimates. The purpose is to compare efficiency of 5 normalization methods with each other.

Simple Additive Weighting (SAW) method was applied to determine the best alternative. Suppose, we have malternatives evaluated according ton criteria and decision making matrix is $\left(r_{i j}\right)_{m \times n}$. Decision making matrix after normalization procedure is $\left(\widetilde{r_{l j}}\right)_{m \times n}$. Let $w_{j}, j=1,2, \ldots, n$ be criteria weights, 
Natalja Kosareva, Aleksandras Krylovas, Edmundas Kazimieras Zavadskas

usually evaluated by experts or calculated by objective methods. The best alternative has the biggest overall aggregated value $Q_{i}=\sum_{j=1}^{n} w_{j} \widetilde{l_{l}}$.

The overview of the most often used normalization methods as well as normalization formulas is given in the Table 1. Data are normalized by applying some monotonic function of initial data matrix elements, which gains its values in the interval $[0,1]$. If preferable values of criteria are bigger, this function is nondecreasing (direct normalization), if preferable values are lower - non-increasing (inverse normalization). As a result, normalized values of all criteria are benefit type, i.e. their greater values are better.

Table 1. Formulas for various normalization methods in the cases of direct and inverse normalization

\begin{tabular}{|c|c|c|}
\hline Normalization method & Direct normalization & Inverse normalization \\
\hline $\begin{array}{l}\text { Vector normalization } \\
\text { (Van Delft and } \\
\text { Nijkamp, 1977) }\end{array}$ & $\widetilde{r_{l j}}=\frac{r_{i j}}{\sqrt{\sum_{i=1}^{m} r_{i j}^{2}}}$ & $\widetilde{r_{l j}}=1-\frac{r_{i j}}{\sqrt{\sum_{i=1}^{m} r_{i j}^{2}}}$ \\
\hline $\begin{array}{l}\text { Max normalization } \\
\text { (Stopp, 1975) }\end{array}$ & $\widetilde{r_{l j}}=\frac{r_{i j}}{\max _{1 \leq i \leq m} r_{i j}}$ & $\widetilde{r_{l j}}=\frac{\min _{1 \leq i \leq m} r_{i j}}{r_{i j}}$ \\
\hline $\begin{array}{l}\text { Sum normalization } \\
\text { (Wang and Luo, 2010) }\end{array}$ & $\widetilde{r_{l j}}=\frac{r_{i j}}{\sum_{i=1}^{m} r_{i j}}$ & $\widetilde{r_{l j}}=\frac{\left(1 / r_{i j}\right)}{\sum_{i=1}^{m}\left(1 / r_{i j}\right)}$ \\
\hline $\begin{array}{l}\text { Logarithmic } \\
\text { normalization } \\
\text { (Zavadskas } \\
\text { Turskis, 2008) }\end{array}$ & $\widetilde{r_{\imath \jmath}}=\frac{\ln \left(r_{i j}\right)}{\ln \left(\prod_{i=1}^{m} r_{i j}\right)}$ & $\widetilde{r_{l \jmath}}=\frac{\left(1-\frac{\ln \left(r_{i j}\right)}{\ln \left(\prod_{i=1}^{m} r_{i j}\right)}\right)}{m-1}$ \\
\hline $\begin{array}{l}\text { Minmax normalization } \\
\text { (Weitendorf , 1976) }\end{array}$ & $\tilde{r}_{i j}=\frac{r_{i j}-\min _{1 \leq i \leq m} r_{i j}}{\max _{1 \leq i \leq m} r_{i j}-\min _{1 \leq i \leq m} r_{i j}}$ & $\tilde{r}_{i j}=\frac{\max _{1 \leq i \leq m} r_{i j}-r_{i j}}{\max _{1 \leq i \leq m} r_{i j}-\min _{1 \leq i \leq m} r_{i j}}$ \\
\hline
\end{tabular}

Numerical experiments were carried out by Monte Carlo method. Initial decision making matrices randomly generated in two ways - when there are more and less separable alternatives. In both cases matrices were generated so, that the first alternative has the higher probability of obtaining better values - higher for the benefit criteria and lower for the cost criteria. Let values of matrices elements be ternary, i.e. they can only get three values $-1,2,3$. The number of alternatives as well as the number of criteria varied from 3 to 5 .

Case 1 . This case reflects situation when the alternatives are more separable. 
Statistical Analysis of MCDM Data Normalization Methods Using Monte Carlo Approach. The Case of Ternary Estimates Matrix

\section{Direct optimization.}

$m=3$ : alternative 1 gains value 3 with probability $p=0.9,0.8,0.7,0.6$, values 1,2 with equal probabilities $\frac{1-p}{2}$. Alternative 2 gains value 2 with probability $p$, values 1 , 3 with equal probabilities. Alternative 3 gains value 1 with probability $p$, values 2 , 3 with equal probabilities.

$m=4$ : alternatives $1,2,4$ are generated in the same manner as the alternatives 1 , 2,3 in the case of 3 alternatives, alternative 3 gains values $1,2,3$ with equal probabilities $(1 / 3)$.

$m=5$ : alternative 1 is generated in the same manner as the alternative 1 , alternatives 2 and 3 - as the alternative 2, alternatives 4 and 5 - as the alternative 3 in the case of 3 alternatives.

Inverse optimization. The random matrix generation process is the same, only values 1 and 3 are replaced their places.

In the Table 2 examples of decision making matrices generated for $n=3, p=$ $0.8, m=3,4,5$ proposed for direct and inverse normalizations.

Table 2. Case 1. Examples of initial decision making matrices in the cases of direct and inverse optimization, $n=3, m=3,4,5, p=0.8$.

\begin{tabular}{|l|c|c|c|}
\hline & $m=3$ & $m=4$ & $m=5$ \\
\hline $\begin{array}{l}\text { Direct } \\
\text { optimization }\end{array}$ & $\left(\begin{array}{lll}3 & 3 & 2 \\
2 & 2 & 2 \\
1 & 2 & 1\end{array}\right)$ & $\left(\begin{array}{lll}2 & 3 & 3 \\
2 & 2 & 2 \\
2 & 1 & 3 \\
1 & 3 & 1\end{array}\right)$ & $\left(\begin{array}{lll}3 & 3 & 2 \\
1 & 2 & 2 \\
2 & 1 & 2 \\
1 & 3 & 1 \\
1 & 1 & 1\end{array}\right)$ \\
\hline $\begin{array}{l}\text { Inverse } \\
\text { optimization }\end{array}$ & $\left(\begin{array}{lll}1 & 1 & 2 \\
2 & 2 & 2 \\
3 & 3 & 3\end{array}\right)$ & $\left(\begin{array}{lll}1 & 1 & 1 \\
1 & 2 & 2 \\
1 & 3 & 1 \\
3 & 3 & 2\end{array}\right)$ & $\left(\begin{array}{lll}2 & 1 & 1 \\
2 & 2 & 2 \\
2 & 3 & 2 \\
3 & 3 & 3 \\
3 & 2 & 3\end{array}\right)$ \\
\hline
\end{tabular}

Case 2. The case reflects situation when the alternatives are less separable.

\section{Direct optimization.}

Alternative 1 gains value 2 with probability $p=0.9,0.8,0.7,0.6$, values 1,3 with equal probabilities $\frac{1-p}{2}$. The other alternatives gain value 1 with probability $p$, values 2, 3 with equal probabilities. 
Natalja Kosareva, Aleksandras Krylovas, Edmundas Kazimieras Zavadskas

Inverse optimization.

Alternative 1 gains value 2 with probability $p=0.9,0.8,0.7,0.6$, values 1 , 3 with equal probabilities $\frac{1-p}{2}$. The other alternatives gain value 3 with probability $p$ values 1, 2 with equal probabilities.In the Table 3 examples of decision making matrices generated for $n=4, p=0.8, m=3,4,5$ proposed for direct and inverse normalizations.

Table 3. Case 2. Examples of initial decision making matrices in the cases of direct and inverse optimization, $n=4, m=3,4,5, p=0.8$.

\begin{tabular}{|l|c|cc|c|c|}
\hline & $m=3$ & $m=4$ & \multicolumn{3}{c|}{$m=5$} \\
\hline $\begin{array}{l}\text { Direct } \\
\text { optimization }\end{array}$ & $\left(\begin{array}{llll}2 & 3 & 2 & 2 \\
1 & 1 & 1 & 1 \\
1 & 1 & 3 & 1\end{array}\right)$ & $\left(\begin{array}{llll}1 & 2 & 2 & 3 \\
1 & 1 & 3 & 1 \\
3 & 3 & 1 & 1 \\
1 & 1 & 2 & 1\end{array}\right)$ & $\left(\begin{array}{llll}2 & 2 & 2 & 2 \\
1 & 1 & 1 & 1 \\
1 & 2 & 1 & 1 \\
3 & 3 & 1 & 1 \\
1 & 1 & 1 & 3\end{array}\right)$ \\
\hline $\begin{array}{l}\text { Inverse } \\
\text { optimization }\end{array}$ & $\left(\begin{array}{llll}2 & 2 & 2 & 1 \\
3 & 3 & 1 & 1 \\
3 & 2 & 3 & 3\end{array}\right)$ & $\left(\begin{array}{llll}2 & 2 & 2 & 3 \\
3 & 1 & 3 & 3 \\
1 & 3 & 3 & 3 \\
3 & 3 & 3 & 3\end{array}\right)$ & $\left(\begin{array}{llll}3 & 2 & 2 & 2 \\
1 & 3 & 3 & 3 \\
3 & 1 & 3 & 3 \\
3 & 3 & 2 & 3 \\
3 & 3 & 3 & 3\end{array}\right)$ \\
\hline
\end{tabular}

\section{A detailed description of the numerical experiments}

100 Monte Carlo experiments were conducted for Case 1 and Case 2 and for such combinations of initial parameters: $m=3,4,5, \quad n=3,4,5, \quad p=$ $0.9,0.8,0.7,0.6$ overally 100 series of 144 experiments.In each experiment, decision making matrices were generated as described in Section 2, matrices elements are normalized in 5 ways according to the formulas given in the Table 1. Then SAW method aggregation formula with equal weights applied and values $Q_{i}$ calculated for each alternative: $Q_{i}=\sum_{j=1}^{n} \frac{1}{n} \tilde{r}_{i j}$. Finally, alternatives are ranked by ascending order of values $Q_{i}$. Each time we fix the result of $\mathrm{j}$-th experiment $R_{j}, j=$ $1,2, \ldots, 100 . R_{j}=1$, if the corresponding method detected alternative 1 as the best one and $R_{j}=0$, otherwise. The number of identification of the first alternative as the best after 100 experiments is $I D=\sum_{j=1}^{100} R_{j} . I D$ ist he measure of identification accuracy. Our purpose is to compare identification accuracy of5 different normalization methods and different parameters $m, n, p$ values. The other calculated value is standard deviation of experiment results $R_{j}$ calculated from 100 Monte Carlo experiments:

$$
S T D=\sqrt{\frac{1}{99} \sum_{j=1}^{100}\left(R_{j}-\bar{R}\right)^{2}},
$$


Statistical Analysis of MCDM Data Normalization Methods Using Monte Carlo Approach. The Case of Ternary Estimates Matrix

Table 4. Case 1. Numbers of the best alternative detection accuracy $(I D)$ and standard deviations of experiment results $($ ST D)for 5 different normalization methods and $\boldsymbol{p}=0.7$.

\begin{tabular}{|c|c|c|c|c|c|c|c|c|c|}
\hline$m=3$ & \multicolumn{3}{|c|}{$n=3$} & \multicolumn{3}{|c|}{$n=4$} & \multicolumn{3}{|c|}{$n=5$} \\
\hline \multirow{6}{*}{$\begin{array}{l}\text { Direct } \\
\text { optimizat } \\
\text { ion }\end{array}$} & & ID & STD & & ID & STD & & ID & STD \\
\hline & Vector & 81 & 0.419 & Vector & 83 & 0.443 & Vector & 91 & 0.373 \\
\hline & Max & 83 & 0.388 & Max & 84 & 0.435 & Max & 92 & 0.362 \\
\hline & Sum & 81 & 0.419 & Sum & 82 & 0.449 & Sum & 91 & 0.373 \\
\hline & $\log$ & 74 & 0.468 & $\log$ & 76 & 0.510 & $\log$ & 70 & 0.510 \\
\hline & Minmax & 85 & 0.359 & Minmax & 86 & 0.386 & Minmax & 93 & 0.307 \\
\hline \multirow{6}{*}{$\begin{array}{l}\text { Inverse } \\
\text { optimizat } \\
\text { ion }\end{array}$} & & ID & STD & & ID & STD & & ID & STD \\
\hline & Vector & 80 & 0.562 & Vector & 87 & 0.411 & Vector & 92 & 0.321 \\
\hline & Max & 81 & 0.456 & Max & 87 & 0.377 & Max & 93 & 0.351 \\
\hline & Sum & 81 & 0.484 & Sum & 89 & 0.427 & Sum & 96 & 0.261 \\
\hline & $\log$ & 83 & 0.443 & $\log$ & 89 & 0.458 & $\log$ & 94 & 0.339 \\
\hline & Minmax & 78 & 0.525 & Minmax & 81 & 0.426 & Minmax & 88 & 0.435 \\
\hline$m=4$ & \multicolumn{3}{|c|}{$n=3$} & \multicolumn{3}{|c|}{$n=4$} & \multicolumn{3}{|c|}{$n=5$} \\
\hline \multirow{6}{*}{$\begin{array}{l}\text { Direct } \\
\text { optimizat } \\
\text { ion }\end{array}$} & & ID & STD & & ID & STD & & ID & STD \\
\hline & Vector & 69 & 0.822 & Vector & 77 & 0.734 & Vector & 79 & 0.566 \\
\hline & Max & 74 & 0.780 & Max & 79 & 0.680 & Max & 81 & 0.557 \\
\hline & Sum & 70 & 0.821 & Sum & 77 & 0.734 & Sum & 79 & 0.566 \\
\hline & $\log$ & 58 & 0.859 & $\log$ & 65 & 0.822 & $\log$ & 66 & 0.655 \\
\hline & Minmax & 75 & 0.718 & Minmax & 81 & 0.674 & Minmax & 83 & 0.524 \\
\hline \multirow{6}{*}{$\begin{array}{l}\text { Inverse } \\
\text { optimizat } \\
\text { ion }\end{array}$} & & ID & STD & & ID & STD & & ID & STD \\
\hline & Vector & 75 & 0.702 & Vector & 80 & 0.621 & Vector & 87 & 0.443 \\
\hline & Max & 77 & 0.697 & Max & 84 & 0.492 & Max & 89 & 0.314 \\
\hline & Sum & 76 & 0.746 & Sum & 82 & 0.548 & Sum & 87 & 0.377 \\
\hline & $\log$ & 76 & 0.685 & $\log$ & 82 & 0.613 & $\log$ & 87 & 0.377 \\
\hline & Minmax & 73 & 0.657 & Minmax & 77 & 0.591 & Minmax & 86 & 0.420 \\
\hline$m=5$ & \multicolumn{3}{|c|}{$n=3$} & \multicolumn{3}{|c|}{$n=4$} & \multicolumn{3}{|c|}{$n=5$} \\
\hline \multirow{6}{*}{$\begin{array}{l}\text { Direct } \\
\text { optimizat } \\
\text { ion }\end{array}$} & & ID & STD & & ID & STD & & ID & STD \\
\hline & Vector & 73 & 0.870 & Vector & 78 & 0.621 & Vector & 85 & 0.705 \\
\hline & Max & 74 & 0.793 & Max & 82 & 0.653 & Max & 87 & 0.613 \\
\hline & Sum & 71 & 0.834 & Sum & 77 & 0.716 & Sum & 86 & 0.668 \\
\hline & $\log$ & 62 & 0.925 & Log & 65 & 0.834 & $\log$ & 69 & 0.835 \\
\hline & Minmax & 78 & 0.772 & Minmax & 83 & 0.609 & Minmax & 84 & 0.642 \\
\hline \multirow{6}{*}{$\begin{array}{l}\text { Inverse } \\
\text { optimizat } \\
\text { ion }\end{array}$} & & ID & STD & & ID & STD & & ID & STD \\
\hline & Vector & 81 & 0.575 & Vector & 88 & 0.705 & Vector & 87 & 0.473 \\
\hline & Max & 83 & 0.518 & Max & 91 & 0.539 & Max & 90 & 0.418 \\
\hline & Sum & 80 & 0.617 & Sum & 91 & 0.545 & Sum & 89 & 0.427 \\
\hline & $\log$ & 85 & 0.512 & $\log$ & 90 & 0.657 & $\log$ & 88 & 0.403 \\
\hline & Minmax & 77 & 0.714 & Minmax & 84 & 0.653 & Minmax & 82 & 0.649 \\
\hline
\end{tabular}


Natalja Kosareva, Aleksandras Krylovas, Edmundas Kazimieras Zavadskas

here $\bar{R}$ is the average value of random variables $R_{j}, j=1,2, \ldots, 100$. The results of 100 Monte Carlo experiments calculated for Case 1 presented in the Table 4.

Analyzing the results of experiments for the Case 1 we can see that for the direct optimization, $p=0.7$ and all $n$ and $m$ values considered, the highest percent of first alternative identification as the best one was shown by Minmax normalization method, meanwhile the lowest percent - by Log method. The only exception - for $m=5$ and $n=5$ the best is Max normalization. In all mentioned cases the highest $I D$ values were accompanied by the lowest standard deviations of experiment results, respectively, the lowest $I D$ values - by the highest standard deviations.

Experiment results for the inverse optimization did not exhibited such clear trend as for the direct optimization. The only sustainable trend is that $I D$ values are the lowest for Minmax normalization method for all $n$ and $m$ values. For $m=3$ the best normalization methods are Log and Sum, for $m=4-$ Max, while for $m=5-\log$ and Max methods.For obtaining more accurate results, we performed calculations with different $p$ values. Calculations performed with 4 different $p$ values $0.9,0.8,0.7,0.6$. Lagrange polynomials(Waring, 1779) were applied for function $I D$ values interpolation, i.e. calculation of function values in the intermediate points. The formula for Lagrange interpolationpolynomial values for the case of 4 points is:

$$
\begin{gathered}
f(p)=f\left(p_{1}\right) \frac{\left(p-p_{2}\right)\left(p-p_{3}\right)\left(p-p_{4}\right)}{\left(p_{1}-p_{2}\right)\left(p_{1}-p_{3}\right)\left(p_{1}-p_{4}\right)} \\
+f\left(p_{2}\right) \frac{\left(p-p_{1}\right)\left(p-p_{3}\right)\left(p-p_{4}\right)}{\left(p_{2}-p_{1}\right)\left(p_{2}-p_{3}\right)\left(p_{2}-p_{4}\right)} \\
+f\left(p_{3}\right) \frac{\left(p-p_{1}\right)\left(p-p_{2}\right)\left(p-p_{4}\right)}{\left(p_{3}-p_{1}\right)\left(p_{3}-p_{2}\right)\left(p_{3}-p_{4}\right)}+f\left(p_{4}\right) \frac{\left(p-p_{1}\right)\left(p-p_{2}\right)\left(p-p_{3}\right)}{\left(p_{4}-p_{1}\right)\left(p_{4}-p_{2}\right)\left(p_{4}-p_{3}\right)} .
\end{gathered}
$$

Variation dynamics of $I D$ depending on the probability $p$ values for the direct optimization in Case 1 is reflected in Figure 1. The trend that the lowest $I D$ values acquired in the case of Log normalization method remains very strong, while the highest $I D$ values most commonly obtained with Minmax and Max normalization. In Figure 2 dependency of $I D$ on the probability $p$ values for the inverse optimization is depicted. On the contrary, in most cases the lowest $I D$ values acquired for Minmax normalization method. The highest $I D$ values generally reached for Max and Log methods, but this trend is not predominant.

The same calculations were performed for the Case 2 when alternatives are less separable, $p=0.7, m=3,4,5$. Monte Carlo experiment results for the Case 2 presented in the Table 5. For the direct optimization one trend is obvious - the highest number of correct detections of the best alternative is observed for Log and Minmax normalization methods, the lowest - for Sum and Max normalizations. The lowest values of standard deviation (STD) also correspond the most accurate normalization methods Log and Minmax, the highest STD values most often are in 
Statistical Analysis of MCDM Data Normalization Methods Using Monte Carlo Approach. The Case of Ternary Estimates Matrix

the cases of Sum and Max normalizations. Inverse optimization has sustainable trend of being Minmax the most accurate normalization method and as a rule (with only one exception) Log is the least accurate normalization method. The least STD values go with Minmax normalization.

Dynamics of IDdepending on the probability $p$ values for the direct optimization in the Case 2 is depicted in Figure 3, for the inverse optimization - in the Figure 4. The assumption that Minmax and Log normalizations are the most exact for the Case 2 and direct optimization has been confirmed. For inverse optimization the most accurate results are always for Minmax normalization, meanwhile the least precise results obtained for Log and Sum normalization methods.

\section{Conclusions and future research}

The purpose of this article is to ascertain how various data normalization methods affect the accuracy of MCDM problem solution. In this research 5 data normalization methods were compared with each other for the solution of MCDM problems of different dimensions and different optimization directions. Case 1, when the alternatives are more separable and Case 2, with the less separable alternatives, were considered. For these cases different scenarios of data matrices random generation were adjusted. In all conducted Monte Carlo experiments decision making matrices were generated with the first alternative as the best one with correspondent probabilitiy $p=0.9,0.8,0.7,0.6$. Then the alternatives were ranked by the SAW method overall aggregated value with equal weights. The measure of identification accuracy $I D$ is the number of identifications of the first alternative as the best one in 100 experiments, i.e. the percentage of correct identifications. Sustainable trends revealed during the experiment are as follows.

Case 1. Direct optimization. The lowest IDvalues obtainedfor the Log normalization.

Case 1. Inverse optimization. In most cases the lowest $I D$ values obtained for the Minmax normalization.

Case 2. Direct optimization. The highest $I D$ values detected for the Log and Minmax normalizations.

Case 2. Inverse optimization. The highest $I D$ values were reached for Minmax normalization, the lowest - for Log and Sum normalization methods.

There were not quite clear tendencies. None of the 5 methods are the best or the worst in all cases. Minmax method in some cases (direct optimization, Case $1, m=3,4)$ is significantly better than other. However, in some cases it is the worst. The Log method is the worst in some cases (Figure 1), but it is the best (or one of the best) in other cases (Figure 2). In some situations efficiency of 5 methods. 
Natalja Kosareva, Aleksandras Krylovas, Edmundas Kazimieras Zavadskas

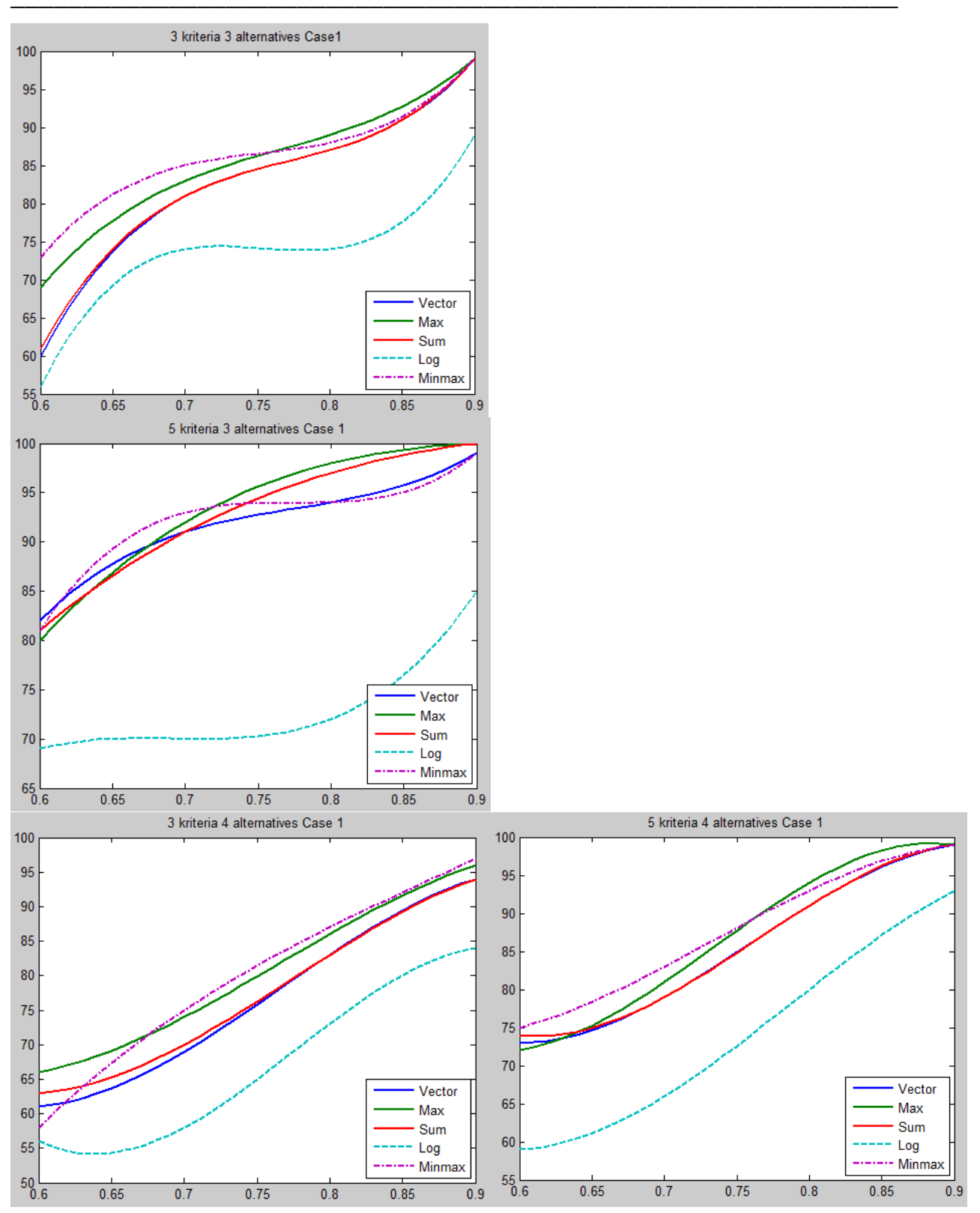


Statistical Analysis of MCDM Data Normalization Methods Using Monte Carlo Approach. The Case of Ternary Estimates Matrix
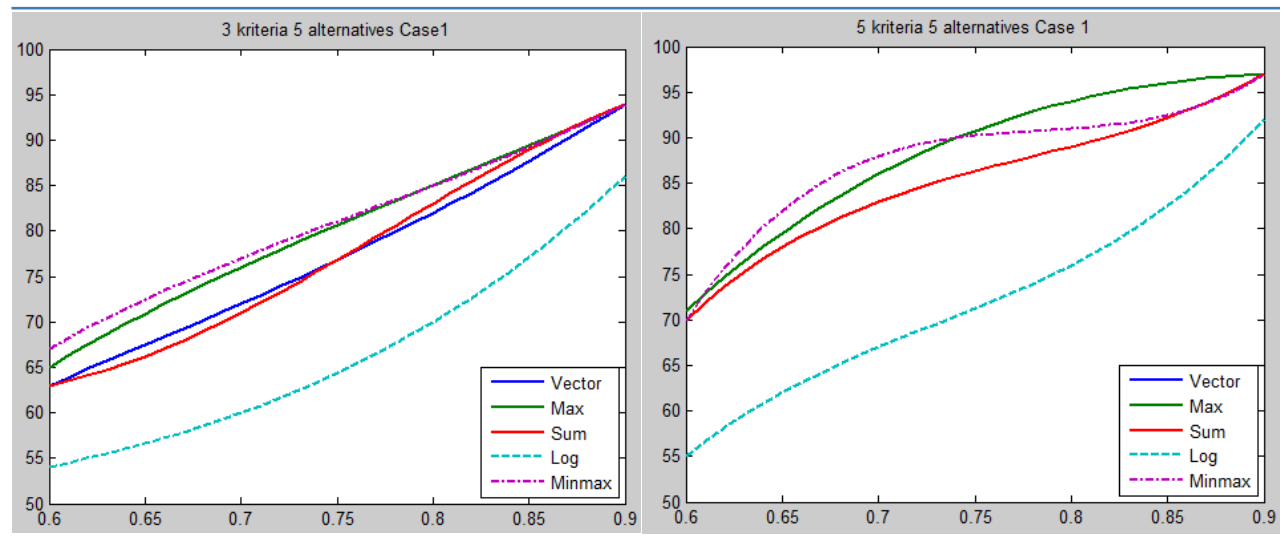

Figure 1. Case 1. Direct optimization. Dependency of detection accuracy (ID) on $p$ values and normalization method $m=3,4,5, n=3,5$.
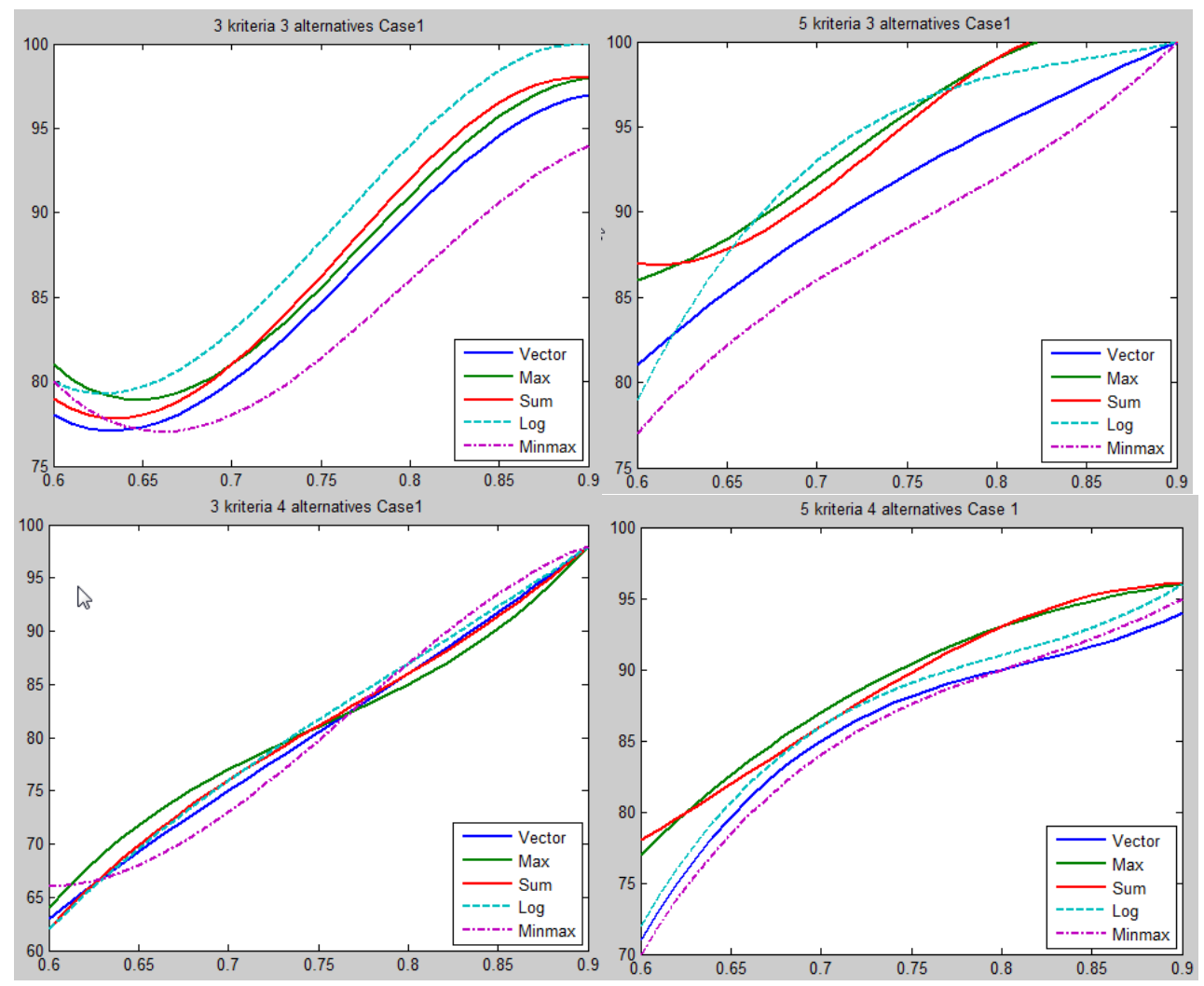
Natalja Kosareva, Aleksandras Krylovas, Edmundas Kazimieras Zavadskas

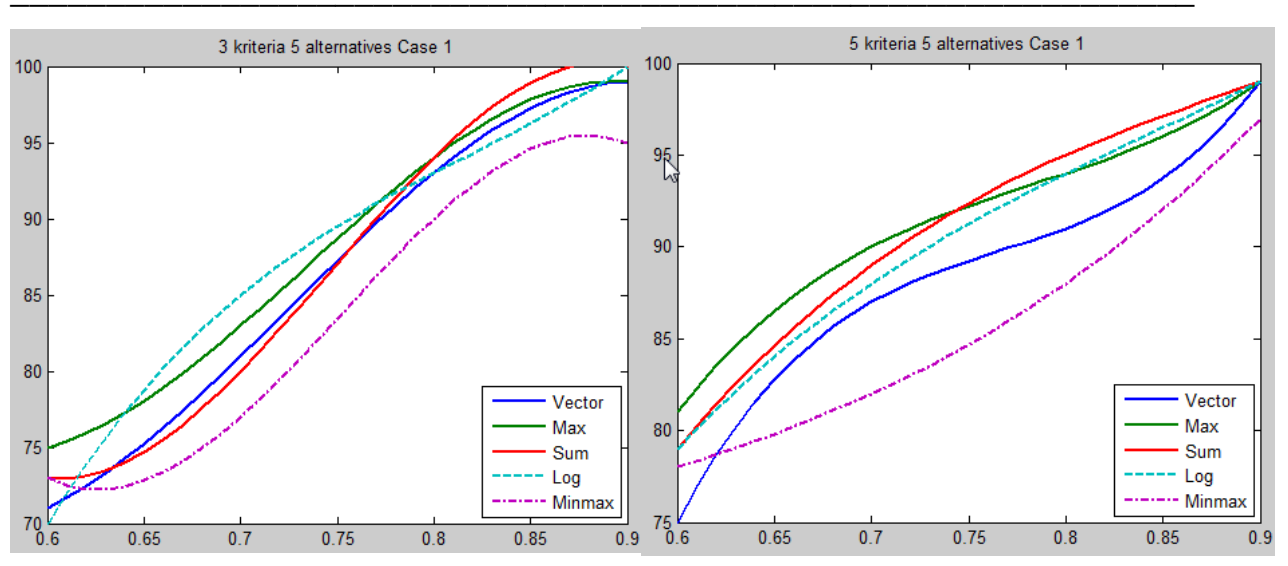

Figure 2. Case 1. Inverse optimization. Dependency of detection accuracy (ID) on $p$ values and normalization $\operatorname{method} m=3,4,5, n=3,5$. 
Statistical Analysis of MCDM Data Normalization Methods Using Monte Carlo Approach. The Case of Ternary Estimates Matrix

Table 5. Case 2. Numbers of the best alternative detection accuracy (ID)and standard deviations of experiment rezults $(S T D)$ for 5 different normalization methods and $\boldsymbol{p}=\mathbf{0 . 7}$.

\begin{tabular}{|c|c|c|c|c|c|c|c|c|c|}
\hline$m=3$ & \multicolumn{3}{|c|}{$n=3$} & \multicolumn{3}{|c|}{$n=4$} & \multicolumn{3}{|c|}{$n=5$} \\
\hline \multirow{6}{*}{$\begin{array}{l}\text { Direct } \\
\text { optimization }\end{array}$} & & ID & STD & & ID & STD & & ID & STD \\
\hline & Vector & 78 & 0.548 & Vector & 82 & 0.386 & Vector & 90 & 0.345 \\
\hline & Max & 75 & 0.514 & Max & 85 & 0.359 & Max & 89 & 0.356 \\
\hline & Sum & 76 & 0.556 & Sum & 82 & 0.419 & Sum & 89 & 0.356 \\
\hline & $\log$ & 89 & 0.458 & $\log$ & 90 & 0.302 & $\log$ & 93 & 0.351 \\
\hline & Minmax & 81 & 0.510 & Minmax & 87 & 0.338 & Minmax & 91 & 0.333 \\
\hline \multirow{6}{*}{$\begin{array}{l}\text { Inverse } \\
\text { optimization }\end{array}$} & & & & & & & & & \\
\hline & Vector & 72 & 0.570 & Vector & 74 & 0.563 & Vector & 83 & 0.471 \\
\hline & Max & 74 & 0.563 & Max & 76 & 0.556 & Max & 82 & 0.449 \\
\hline & Sum & 66 & 0.638 & Sum & 70 & 0.632 & Sum & 76 & 0.533 \\
\hline & $\log$ & 54 & 0.699 & Log & 62 & 0.704 & Log & 71 & 0.648 \\
\hline & Minmax & 92 & 0.273 & Minmax & 88 & 0.435 & Minmax & 93 & 0.351 \\
\hline$m=4$ & \multicolumn{3}{|c|}{$n=3$} & \multicolumn{3}{|c|}{$n=4$} & \multicolumn{3}{|c|}{$n=5$} \\
\hline \multirow{6}{*}{$\begin{array}{l}\text { Direct } \\
\text { optimization }\end{array}$} & & ID & STD & & ID & STD & & ID & STD \\
\hline & Vector & 78 & 0.548 & Vector & 79 & 0.515 & Vector & 87 & 0.411 \\
\hline & Max & 75 & 0.514 & Max & 80 & 0.534 & Max & 85 & 0.428 \\
\hline & Sum & 76 & 0.556 & Sum & 78 & 0.520 & Sum & 87 & 0.411 \\
\hline & $\log$ & 89 & 0.458 & $\log$ & 90 & 0.383 & $\log$ & 93 & 0.256 \\
\hline & Minmax & 81 & 0.510 & Minmax & 89 & 0.356 & Minmax & 91 & 0.333 \\
\hline \multirow{6}{*}{$\begin{array}{l}\text { Inverse } \\
\text { optimization }\end{array}$} & & ID & STD & & ID & STD & & ID & STD \\
\hline & Vector & 57 & 0.755 & Vector & 74 & 0.541 & Vector & 75 & 0.598 \\
\hline & Max & 55 & 0.800 & Max & 72 & 0.626 & Max & 72 & 0.626 \\
\hline & Sum & 44 & 0.811 & Sum & 57 & 0.716 & Sum & 63 & 0.624 \\
\hline & Log & 44 & 0.877 & Log & 53 & 0.817 & $\log$ & 57 & 0.793 \\
\hline & Minmax & 79 & 0.562 & Minmax & 89 & 0.356 & Minmax & 91 & 0.433 \\
\hline$m=5$ & \multicolumn{3}{|c|}{$n=3$} & \multicolumn{3}{|c|}{$n=4$} & \multicolumn{3}{|c|}{$n=5$} \\
\hline \multirow{6}{*}{$\begin{array}{l}\text { Direct } \\
\text { optimization }\end{array}$} & & ID & STD & & ID & STD & & ID & STD \\
\hline & Vector & 68 & 0.728 & Vector & 72 & 0.659 & Vector & 79 & 0.640 \\
\hline & Max & 71 & 0.661 & Max & 77 & 0.591 & Max & 77 & 0.548 \\
\hline & Sum & 68 & 0.699 & Sum & 71 & 0.661 & Sum & 77 & 0.647 \\
\hline & $\log$ & 78 & 0.587 & $\log$ & 83 & 0.543 & $\log$ & 87 & 0.526 \\
\hline & Minmax & 80 & 0.597 & Minmax & 85 & 0.506 & Minmax & 88 & 0.403 \\
\hline \multirow{6}{*}{$\begin{array}{l}\text { Inverse } \\
\text { optimization }\end{array}$} & & ID & STD & & ID & STD & & ID & STD \\
\hline & Vector & 61 & 0.856 & Vector & 59 & 0.842 & Vector & 65 & 0.671 \\
\hline & Max & 61 & 0.990 & Max & 61 & 0.948 & Max & 55 & 0.801 \\
\hline & Sum & 44 & 1.053 & Sum & 47 & 1.014 & Sum & 49 & 0.830 \\
\hline & $\log$ & 51 & 1.058 & $\log$ & 42 & 1.010 & $\log$ & 45 & 0.947 \\
\hline & Minmax & 75 & 0.634 & Minmax & 82 & 0.524 & Minmax & 86 & 0.420 \\
\hline
\end{tabular}


Natalja Kosareva, Aleksandras Krylovas, Edmundas Kazimieras Zavadskas

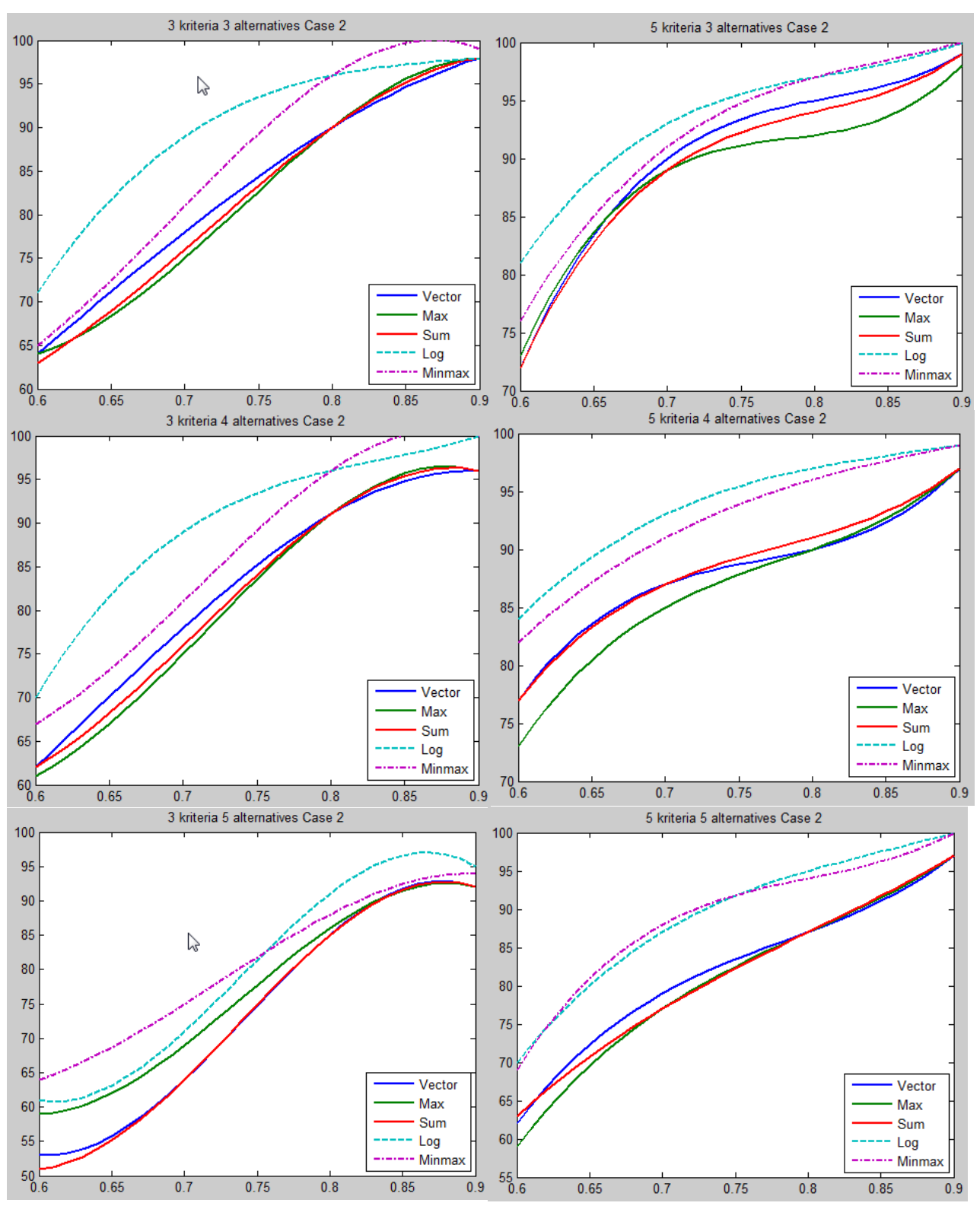

Figure 3. Case 2. Direct optimization. Dependency of detection accuracy (ID) on $p$ values and normalization method $m=3,4,5, n=3,5$. 
Statistical Analysis of MCDM Data Normalization Methods Using Monte Carlo Approach. The Case of Ternary Estimates Matrix
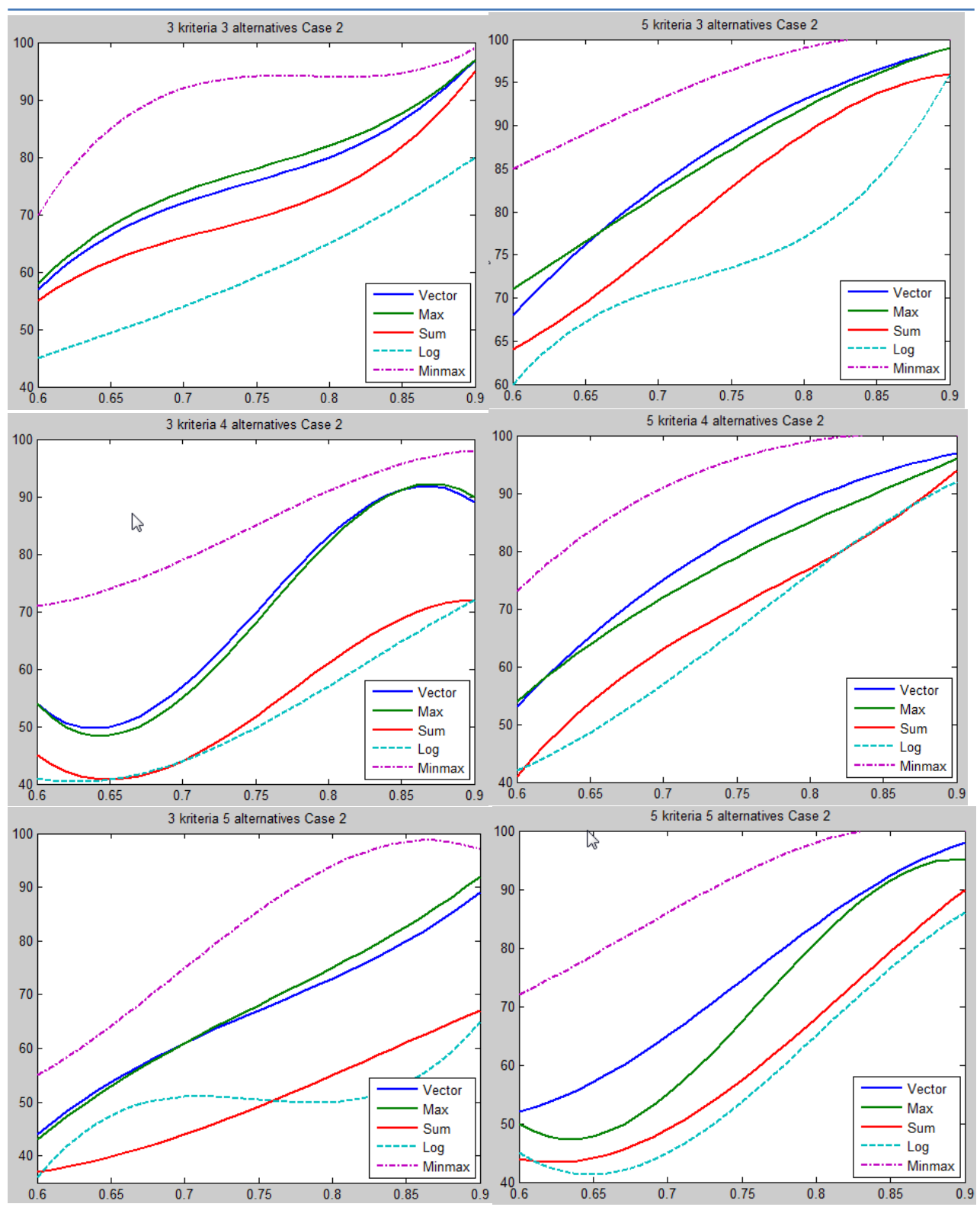

Figure 4. Case 2. Inverse optimization. Dependency of detection accuracy (ID) on $p$ values and normalization method $m=3,4,5, n=3,5$. 
Natalja Kosareva, Aleksandras Krylovas, Edmundas Kazimieras Zavadskas

significantly differ (Figure 4), meanwhile in other cases their efficienciy is very similar. In most cases the highest $I D$ values were accompanied by the lowest standard deviations of experiment results, respectively, the lowest IDvalues - by the highest standard deviations. All results were received dealing with ternary estimates matrices and two cases of their probability distributions. So, the conclusions are preliminary and do not allow to formulate practical recommendations. A more precise establishing of generated matrices would allow the formulation of such recommendations. This requires additional research that is planned for the future.

\section{REFERENCES}

[1] Celen, A. (2014), Comparative Analysis of Normalization Procedures in TOPSIS Method: With an Application to Turkish Deposit Banking

Market.Informatica 25, 2, 185-208;

[2] Chakraborty, S. and Yeh, C.-H. (2009), A Simulation Comparison of Normalization Procedures for TOPSIS. Published in: 2009 International Conference on Computers \& Industrial Engineering, 6-9 July 2009, Troyes, France;

[3] Jahan, A. and Edwards, K. L. (2015), A State-of-the-Art Survey on the Influence of Normalization Techniques in Ranking: Improving the Materials Selection Process in Engineering Design.Materials \& Design65, 335-342; [4] Kaplinski, O. and Tamošaitienė, J. (2015), Analysis of Normalization Methods Influencing Results: A Review to Honour Professor Friedel Peldschus on the Occasion of his 75th Birthday.Procedia Engineering 122:2-10;

[5] Krylovas, A., Kosareva, N. and Zavadskas, E. K. (2017), WEBIRA Comparative Analysis of Weight Balancing Method.International Journal of Computers Communications \& Control12,2, 238-253;

[6] Krylovas, A., Kosareva, N. and Zavadskas, E. K. (2018), Scheme for Statistical Analysis of Some ParametricNormalization Classes. International Journal of Computers Communications \& Control13,6,972-987;

[7] Opricovic, S. and Tzeng, G.H. (2004),Compromise Solution by MCDM Methods: A Comparative Analysis of VIKOR and TOPSIS.Eur J Oper Res 156, 445-455;

[8] Podviezko, A. and Podvezko, V. (2015), Influence of Data Transformation on Multicriteria Evaluation Result.Procedia Engineering 122, 151-157;

[9] Stanujkic,D., Zavadskas, E. K., Karabasevic, D., Turskis, Z. and Keršulienė, V. (2017a), New Group Decision-Making ARCAS Approach Based on the Integration of the SWARA and the ARAS Methods Adapted for Negotiations.Journal of Business Economics and Management 18, 4, 599-618; [10] Stanujkic, D., Zavadskas, E. K., Liu, S., Karabasevic, D. and Popovic, G. (2017b),Improved OCRA Method Based on the Use of Interval Grey Numbers. Journal of Grey System29, 4,49-60; 
Statistical Analysis of MCDM Data Normalization Methods Using Monte Carlo Approach. The Case of Ternary Estimates Matrix

[11] Stanujkic,D., Zavadskas, E. K. (2015), A Modified Weighted Sum Method Based on the Decision-maker's Preferred Levels of Performances. Studies in Informatics and Control 24, 4, 461-469;

[12] Stopp, F. (1975), Variantenvergleich durch Matrixspiele. Wissenschaftliche Zeitschrift der Hochschule für Bauwesen Leipzig, Heft 2;

[13] Van Delft, A. and Nijkamp, P. (1977), Multi-Criteria Analysis and Regional Decision-Making. M. Nijhoft, Leiden, Nl;

[14] Wang, Y.-M. and Luo, Y. (2010), Integration of Correlations with Standard Deviations for Determining Attribute Weights in Multiple Attribute Decision Making.Mathematical and Computer Modelling51,1-2, 1-12;

[15] Waring, E. (1779),Problems Concerning Interpolations. Philosophical Transactions of the Royal Society69, 59-67;

[16] Weitendorf, D. (1976), Beitrag zur Optimierung der räumlichen Struktur eines Gebäudes.Dissertation A.Hochschule für Architektur und Bauwesen. Weimar;

[17] Yazdani, M., Jahan, A. and Zavadskas, E. K. (2017), Analysis in Material Selection: Influence of Normalization Tools on COPRAS-G. Economic

Computation and Economic Cybernetics Studies and Research 51, 1, 59-74;

[18] Zavadskas, E. K. and Turskis, Z. (2008), A New Logarithmic Normalization Method in Games Theory. Informatica 19, 2, 303-314;

[19] Zavadskas, E. K., Zakarevicius,A. and Antucheviciene, J. (2006), Evaluation of Ranking Accuracy in Multi-criteria Decisions. Informatica 17, 4, 601-618. 